\title{
Upscaling of wood bilayers: design principles for controlling shape change and increasing moisture change rate
}

\author{
C. Vailati $\cdot$ P. Hass $\cdot$ I. Burgert $\cdot$ M. Rüggeberg $(\mathbb{D}$
}

Received: 4 April 2017/ Accepted: 20 November 2017/Published online: 29 November 2017

(C) RILEM 2017

\begin{abstract}
Wood exhibits anisotropic swelling and shrinking upon changes of wood moisture content (MC). By manufacturing bi-layered structures with adapted grain orientation in the two bonded layers, humidity-driven actuators are generated, which have the potential to be used for autonomous climate adaptive building with tile. The present study deals with design principles for upscaling the size of the bilayers and for increasing the rate of MC change and, thus, rate of shape change. Wood bilayers with widths of up to half a meter were subjected to changes of relative humidity (RH). Moisture and curvature changes were recorded. Bilayers with different widths showed curvature exclusively along their length. Next to this, the performance was compared between bilayers with and without milled-in grooves. These grooves lead to shorter diffusion paths along fibre direction for increasing the rate of MC change. The highest rates of MC change were visible for the samples with the
\end{abstract}

Electronic supplementary material The online version of this article (https://doi.org/10.1617/s11527-017-1117-4) contains supplementary material, which is available to authorized users.

C. Vailati · P. Hass · I. Burgert · M. Rüggeberg ( $\square)$ Institute for Building Materials (IfB), ETH Zurich, 8093 Zurich, Switzerland

e-mail: mrueggeberg@ethz.ch

C. Vailati · P. Hass · I. Burgert · M. Rüggeberg Laboratory of Applied Wood Materials, EMPA, 8600 Dübendorf, Switzerland smallest width within the first hours after change of RH. Later on, all samples showed similar rates. The milling of grooves increased the moisture change rate substantially compared to the non-milled samples resulting in a higher rate of curvature change. The increase is especially pronounced for cyclic changes of RH. This study shows that, by applying material specific design principles, the shape change of wood bilayers can be adapted and the rate of the MC change can be increased by keeping diffusion paths short along fibre direction. These principles may facilitate the use of large-scale wood bilayers as lamellae in shading systems.

Keywords Wood bilayer - Smart material · Upscaling · Climate adaptive building shells . Autonomous responsiveness

\section{Introduction}

Since the adoption of the Kyoto Protocol, energy efficiency and energy savings have become central emerging topics. In particular, the building sector, with heating, cooling and ventilation systems, is one of the major consumers of energy [1]. Therefore, many architectural solutions have been developed for reducing the energy consumption of buildings. Systems like the Trombe wall or the solar chimney store and 
use solar and wind energy. Next to this, climate adaptive building shells [2] have been developed, which respond to changes of outside climatic conditions by changing configuration or shape. Inspired by plant movements in nature, smart materials have been developed, which can transform the exterior part of the building into an active skin [3]. Mechanical actuators have been fabricated, which specifically respond to different stimuli such as electrical [4], chemical [5], thermal, acoustic, optical [6] or hygro-thermal [7]. These actuators are simple, energy efficient, mutable and evolvable.

In nature, the opening and closing of pine cones or the movement of wheat awns are based on the change of relative humidity $(\mathrm{RH})$ [8-10]. The scales of the pine cones represent a bilayer structure, as they are made up of two connected tissue layers. The two layers show differential orientation distribution of cellulose microfibrils in the cell walls of the respective layers. The orientation pattern of the cellulose fibrils leads to swelling anisotropy and the specific combination of the two layers results in a bending movement and opening of the cone upon drying of such bilayered scales [11, 12]. Inspired by these natural actuators, bilayers were produced with aligned flax fibres embedded into a polymer matrix [13] or by the combination of a plastic sheet and paper [12]. Small wood bilayer strips were fabricated consisting of a layer of beech wood and a layer of spruce wood bonded to each other [14]. Due to the orthogonal fibre direction (grain orientation) in both layers and the swelling coefficient perpendicular to fibre direction (across the grain) being more than one order of magnitude higher than the swelling coefficient parallel (along the grain) to fibre direction, these bilayers bend in response to changes of RH. Rüggeberg and Burgert [14] have used the theory of the bending for bimetal thermostats upon the change of temperature [15] to predict and to optimize the configuration of the wood bilayers and showed, that the amplitude of curvature can be influenced by different factors such as the overall thickness of the bilayer, as well as the thickness ratio of the two layers. Furthermore, two field experiments over 9 months and 1 year, respectively, with the wood bilayers exposed to full weathering, proofed long-term stability of the material, the adhesive and the shape change $[14,16]$. In another field study, actuation of bilayers, which were protected from direct precipitation, was retained for 3 years [17].

So far, hygromorphic wood bilayers have only been studied at small scale exhibiting strip geometry; however, practical applications, e.g. large-scale façade shading systems, require an upscaling of the size of bilayer elements to meter scale. Wood with its unique combination of stiffness, responsiveness and workability, in principle, facilitates the required upscaling; yet, geometric challenges exist for wood bilayers in application-relevant dimensions. Both layers change their dimension perpendicular to fibre direction, which may result in bi-directional, anticlastic (hyperboloid) curvature for larger widths [18]. Hence, for upscaling the size for the targeted application, the bi-directional curvature change needs to be suppressed, in order to achieve a monoclastic (unidirectional) curvature of the actuator. In addition, limitations in the rate of shape change due to the limited rate of diffusion of water in wood [19] may impede a technical implementation, as, e.g., a sufficiently high rate of shape change is needed for an efficient shading system. These challenges and limitations require a thorough understanding of the material physics for elucidating possibilities of optimization.

In this study, we demonstrate actuation of largescale wood bilayers and introduce design principles for, firstly, retaining monoclastic curvature rather than hyperboloid curvature of such upscaled bilayers upon changes of RH. Secondly, we show possibilities for increasing the rate of moisture content (MC) change of such bilayers through shortening of diffusion path lengths especially along fibre direction. These principles may facilitate the utilization of large-scale wood bilayers in autonomous, climate-adaptive façade shading systems.

\section{Materials and methods}

\subsection{Bilayer manufacturing}

Wood bilayers were manufactured by bonding a layer of beech wood (Fagus sylvatica) and a layer of spruce wood (Picea abies) with orthogonal orientation of the fibres of the two layers [14]. Hereby, the length of the bilayers was defined as the dimension orthogonal to the fibre direction (across the grain) of the beech layer 


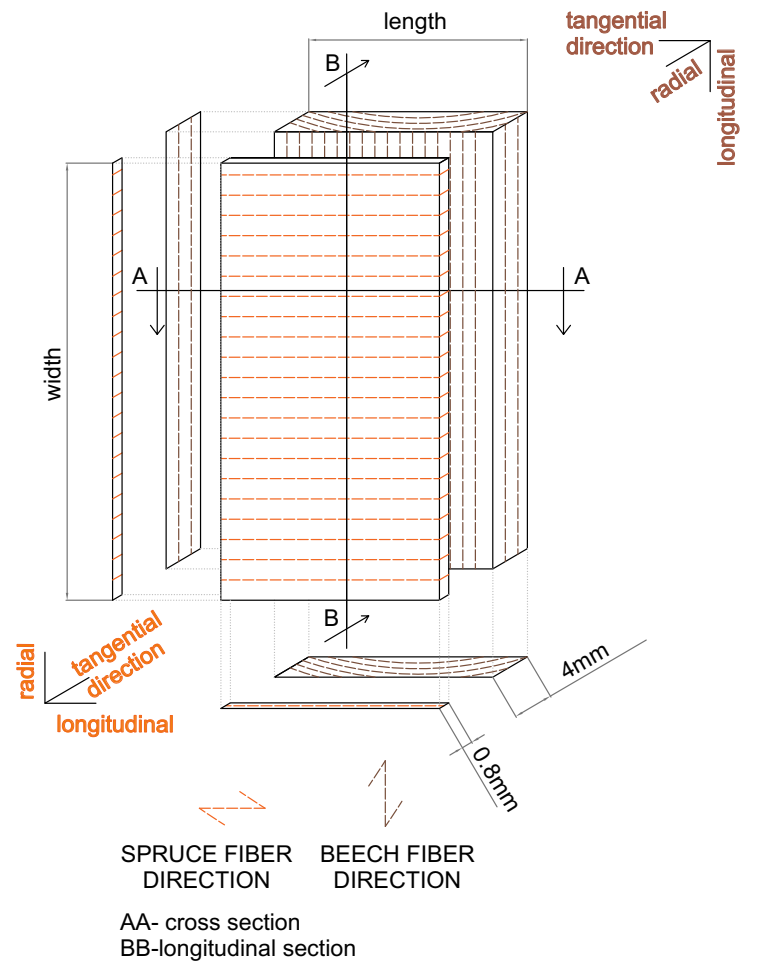

Fig. 1 Setup of a wood bilayer at high $\mathrm{RH}(\sim 85 \%)$ : axonometric projection of the two layers with indicated fibre direction in the beech and spruce layer, definition of length and width and definition of cross-section and longitudinal section

(Fig. 1), meanwhile the width refers to the dimension parallel to the fibre direction (along the grain) of the beech layer. The spruce layer was cut with semi-radial orientation of the annual rings, whereas the beech layer was cut with tangential orientation of the annual rings. The tangential direction for the cutting was chosen to obtain the highest possible dimensional changes along the length of the bilayers (Fig. 1), as swelling in the tangential direction is about twice as high as in the radial direction [20]. The thickness of the beech layer was $4 \mathrm{~mm}$, while the thickness of the spruce layer was $0.8 \mathrm{~mm}$. Therefore, the ratio of thickness was 0.2 to facilitate monoclastic curvature (Online Resource 1-Fig.S1-b). Different sets of bilayers with different sizes were manufactured as described in the following sections. Conditioning and bonding of the wood layers were conducted in a climate chamber at $20{ }^{\circ} \mathrm{C}$ and $85 \% \mathrm{RH}$. The conditioning of the wood prior to the bonding and the distribution of the adhesive (polyurethane adhesive, Purbond HB-S709) on the wood were done according to the manufacturer's recommendations. Before the required pressure for the bonding was applied by loading the samples in a press, the samples were wrapped in a plastic bag in order to minimize the change of MC during pressing.

\subsection{Upscaling the width of bilayers}

Bilayers with a length of $120 \mathrm{~mm}$ and a width of 20 , 40, 80, 120, 240 and $480 \mathrm{~mm}$ were prepared. For each width, five samples were fabricated. Samples were cut from neighboring positions within the same trunk in order to achieve a minimum of natural inhomogeneity of the wood material. After manufacturing, the samples were stored at $20{ }^{\circ} \mathrm{C}$ and $85 \% \mathrm{RH}$. Once the equilibrium $\mathrm{MC}$ had been reached, the samples were moved to a different climate chamber with $20^{\circ} \mathrm{C}$ and $35 \% \mathrm{RH}$ for desorption experiments. The MC loss and change of curvature along the length and width of each sample were analyzed. The weight of the samples was recorded after $1,2,3,4,6,8$, and $24 \mathrm{~h}$ with a scale (precision: 2 decimal digits) for determining the MC [21]. The curvature of the bilayers along the length was monitored by taking images from both sides of each sample using a fixed camera setup. At the same time, the weight was measured. The average curvature was taken for further analysis.

\subsection{Increasing the rate of moisture content change}

Fifteen wood bilayers were fabricated with a length of $140 \mathrm{~mm}$ and a width of $500 \mathrm{~mm}$ and subjected twice to a change of RH from 85 to $35 \%$. Temperature was hereby kept constant at $20{ }^{\circ} \mathrm{C}$. After each desorption phase of $24 \mathrm{~h}$, they were put back to $85 \% \mathrm{RH}$ for reequilibration. Afterwards, the beech layers of 10 samples were modified by milling grooves of $3 \mathrm{~mm}$ depth and $20 \mathrm{~mm}$ width, with a distance of $20 \mathrm{~mm}$ (Fig. 2).

The grooves were milled in two different orientations. For five samples, the orientation of the grooves was parallel to the length of the samples (horizontal configuration) across the grain of the beech layer. For the other five samples, the grooves were milled at an angle of $45^{\circ}$ (diagonal configuration) with respect to the length of the samples (Fig. 2). These milling patterns resulted in a distance of the grooves along fibre direction of $20 \mathrm{~mm}$ for the horizontal configuration and $28 \mathrm{~mm}$ for the diagonal configuration 

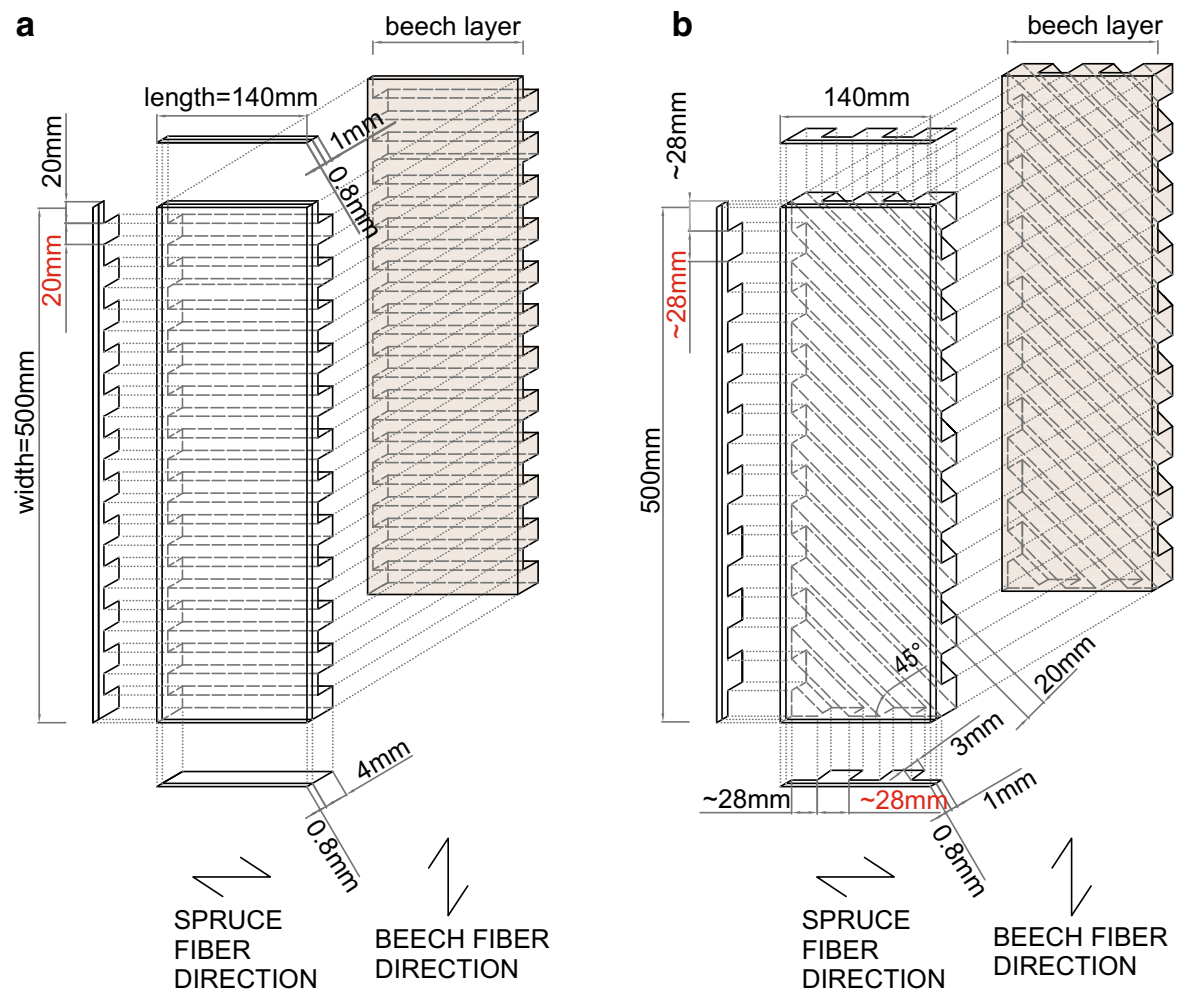

Fig. 2 Axonometric projection at high RH $(\sim 85 \%)$ of bilayers with milled-in grooves with either a horizontal or b diagonal orientation of the grooves

(Fig. 2). The shape change of the 10 modified and the 5 reference samples was then tested in two setups. Firstly, the samples were subjected to an instantaneous change of $\mathrm{RH}$ from 85 to $35 \%$ as described in chapter 2.2. Secondly, three samples from each category were exposed to a cyclic change of RH in a custom-made climate chamber (with a temperature between 20 and $22{ }^{\circ} \mathrm{C}$ ). The samples were fixed with their long edge to a steel frame (Online Resource 1-Fig.S1-a). The steel frame was then put into the climate chamber provided with anti-reflective glass windows for image recording. A ventilation system was installed on the upper and bottom part of the chamber. Dry and wet air were inserted into the chamber from the lower and upper part through a system of sealed plastic tubes in an alternating manner for cyclic alteration of $\mathrm{RH}$. The flow rate was controlled via air pressure. $\mathrm{RH}$ and temperature in the chamber were recorded by two sensors placed in the top and bottom part of the chamber. Cycle times were subsequently decreased from initially $24 \mathrm{~h}$ to 12 ,
6, 4 and finally $2 \mathrm{~h}$. Four loops of desorption/ adsorption cycles were conducted.

In the first setup, weight and curvature along the length were recorded as described for the investigation on upscaling width. In addition to that, also the curvature along the width was quantified by taking images from all four sides of each sample. In the second setup, with the custom-made climate chamber, curvature along the two lengths was recorded with two cameras placed at the same height and the same distance from the climate chamber (Online Resource 1-Fig.S1-a). Images of the two edges along the lengths of all samples were taken by the two computercontrolled cameras every $5 \mathrm{~min}$.

\subsection{Diffusion of water in wood}

Three bilayers consisting of beech wood and aluminum foil were manufactured with $42 \mathrm{~mm}$ length and $96 \mathrm{~mm}$ width (Online Resource 1-Fig.S2-a). Hereby, the aluminum foil acts as a barrier for the diffusion of the water. The samples were cut into nine 
smaller pieces of $13 \mathrm{~mm}$ length and $30 \mathrm{~mm}$ width and stored at $20{ }^{\circ} \mathrm{C}$ and $85 \% \mathrm{RH}$. After equilibration, the sides of the samples were covered with an aluminum tape as shown in Online Resource 1-Fig.S2-b. Based on the fibre direction in the samples and the number of sides sealed with the aluminum foil, the samples were divided into four groups (groups A-D). Sample with three sides covered belong to group A. In this group, only one cross section of the sample, was sealed with the aluminum foil. Samples with four sides covered by aluminum foil belong to groups B and D. In group B, only one cross section was covered while in group $\mathrm{D}$ both cross-sections were covered. Samples with five sides covered by the aluminum foil belong to group $\mathrm{C}$. In this group, both sides cut along the tangential direction were sealed. The bilayers were moved from $85 \% \mathrm{RH}$ to a climate chamber with $50 \% \mathrm{RH}$ (with the temperature kept at $20^{\circ} \mathrm{C}$ ). The weight of all samples was recorded every hour until $8 \mathrm{~h}$ after transfer and at $24 \mathrm{~h}$ after transfer with a scale (3 decimal places). After removing the aluminum foil, all 27 samples were dried in an oven at $103 \pm 5{ }^{\circ} \mathrm{C}$ in order to determine their dry weight.

\subsection{Evaluation of moisture content and curvature}

The MC of the samples was determined from their weights with the double weight method [21]. Hereby, samples are weighted at actual moisture state and at oven-dry state after drying at $103{ }^{\circ} \mathrm{C}$ until weight constancy was reached. The weight difference in relation to the dry weight represents the MC (mass of water related to dry mass of wood). In some cases, reference samples have been taken and dried for evaluating MC. Curvature along the length of the samples was evaluated by painting dots on each investigated edge of each sample prior to taking images of the samples. The images were corrected for lens distortion with Adobe Photoshop Lightroom 6. Using a Matlab R 2015 script, circles were fitted through the coloured dots on the recorded images and curvatures were calculated. The rate of MC loss and the rate of change of curvature were evaluated through a nonlinear curve fitting using a linear combination of two exponential functions and a Levenberg-Marquardt algorithm with then deriving interpolated values for time intervals of one hour. Statistical analysis was done by ANOVA at $95 \%$ confidence level. Furthermore, specific curvature defined as the change of curvature over the change of MC was calculated in order to compare curvature changes in case of differences in MC losses.

\subsection{Simulation of curvature}

Theoretical values of curvature were calculated by adapting Timoshenko's theory of bimetal thermostats (Eq. 1). Hereby, the stiffness ratio $n=E 1 / E 2$ (longitudinal/tangential) was set to 20 according to literature values $[22,23]$. The tangential shrinkage coefficient for beech was set to $0.004 / \%$ while the longitudinal shrinkage coefficient for the spruce layer was set to $0.0001 / \%$ according to Rüggeberg and Burgert [14]. With $h_{1}=0.8 \mathrm{~mm}$ (thickness of the spruce layer) and $h_{2}=4 \mathrm{~mm}$ (thickness of the beech layer), the overall thickness of the bilayer was $h=4.8 \mathrm{~mm}$ and the thickness ratio $\mathrm{m}$ was 0.2 .

$$
\begin{aligned}
& \frac{1}{\rho}=\frac{6(1+m)^{2}}{\left(3(1+m)^{2}+(1+m n)\left(m^{2}+\frac{1}{m n}\right)\right)} \frac{\left(\alpha_{2}-\alpha_{1}\right)\left(c-c_{0}\right)}{h}, \\
& m=\frac{h_{1}}{h_{2}}, n=\frac{E_{1}}{E_{2}}
\end{aligned}
$$

\section{Results}

\subsection{Upscaling width}

Amplitude and rate of moisture content loss as well as amplitude and rate of shape change of wood bilayers with different width were analyzed as a function of the width for revealing the effect of upscaling on these parameters. Next to this, special attention was paid o occurrence of any bi-directional (hyperboloid) curvature as a result of upscaling the width of wood bilayers. At $85 \% \mathrm{RH}$, the beech-spruce bilayers had a $\mathrm{MC}$ of around $18 \%$. Within $48 \mathrm{~h}$ after the transfer to $35 \% \mathrm{RH}$, the MCs decreased to values between 9 and $10 \%$, which corresponds to a relative loss of $\mathrm{MC}$ between 43 and $45 \%$ (Fig. 3a). Differences can clearly be seen for the initial rate of MC loss (Fig. 3c) after the transfer to $35 \%$ RH. The highest value $\left(2.4 \pm 0.1 \% \mathrm{~h}^{-1}\right)$ was obtained for the bilayers with the smallest width $(20 \mathrm{~mm})$ for the time interval between 0 and $1.5 \mathrm{~h}$ (Fig. 3c). The rate of MC loss for the same time interval was also higher for the bilayers 
a

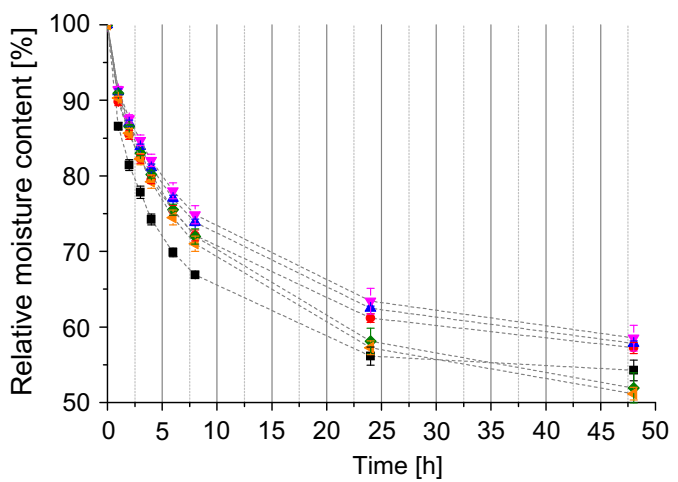

C
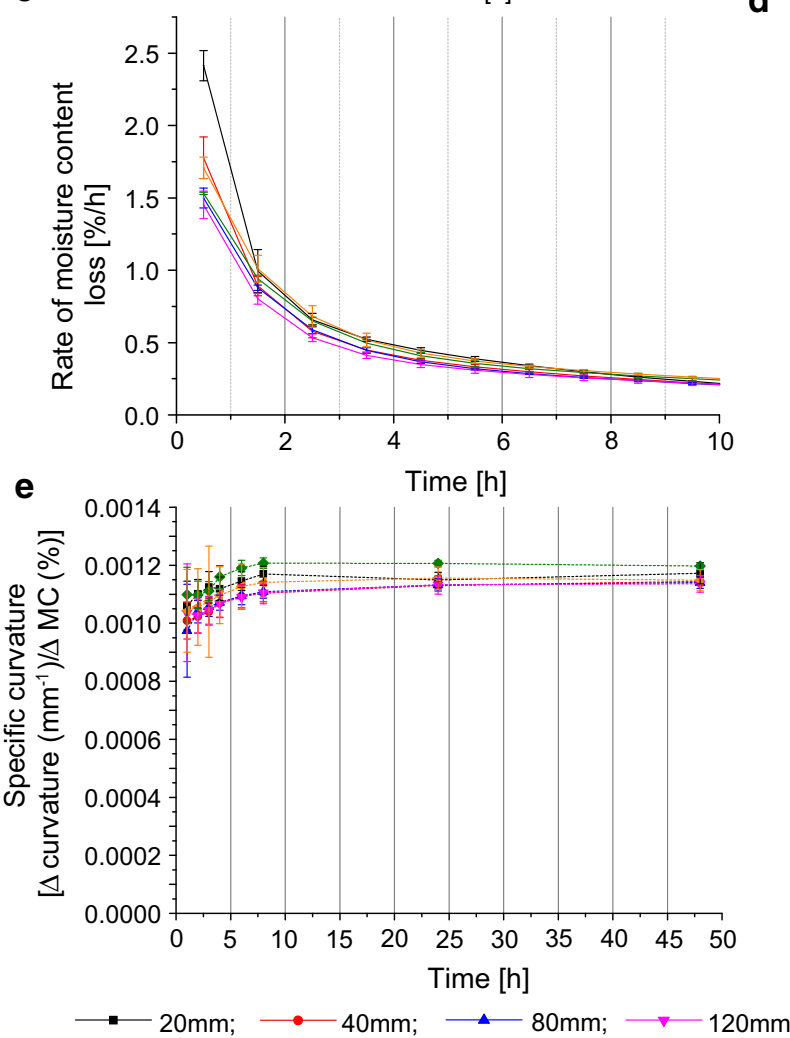

Fig. 3 Change of MC and curvature of bilayers with different width after transfer from 85 to $35 \% \mathrm{RH}$, colour code indicates the different widths. a Relative MC (initial $\mathrm{MC}=100 \%$ ). b Change of curvature along the length (inset: legend for the sign of curvature along the $Y$-axis). $\mathbf{c}$ Rate of the MC loss. The plot values represent the medium values of the nonlinear curve

with $40 \mathrm{~mm}$ width and $480 \mathrm{~mm}$ width compared to the rates of the other configurations.

The loss of moisture content induced curvature of all bilayers perpendicular to the fibre direction of the beech layer, while the bilayers remained straight in fibre direction of the beech layer. Thus, uni-directional

b
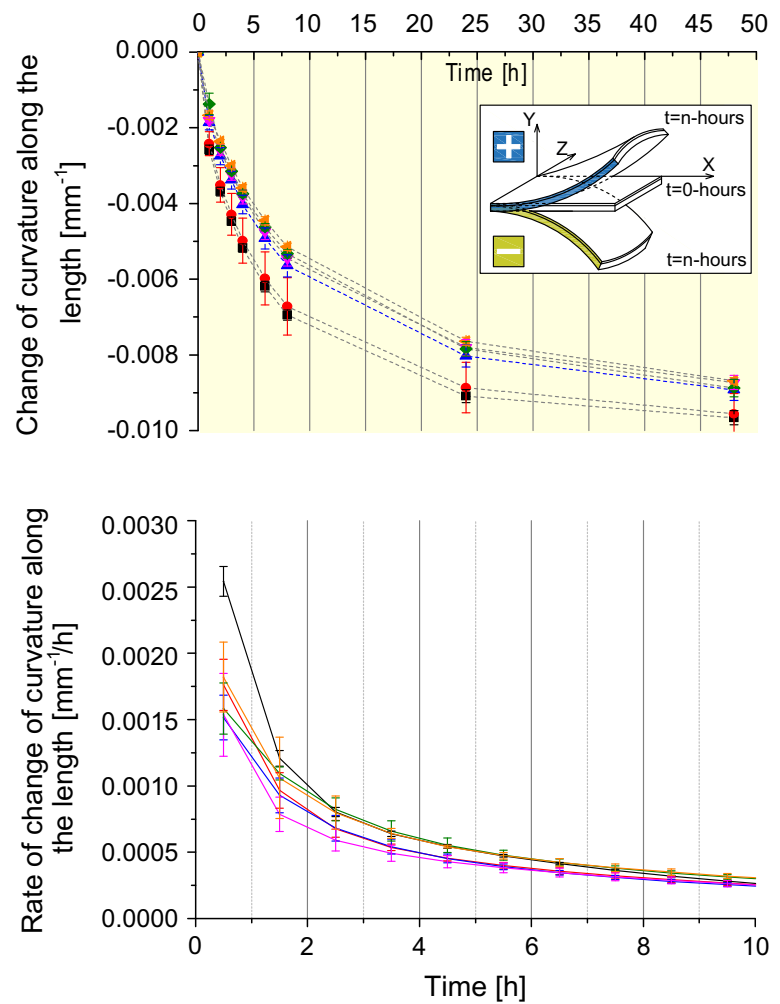

fitting done from the measured data in the first $9.5 \mathrm{~h}$. d Rate of curvature change along the length within the first $9.5 \mathrm{~h}$. Data represent averaged non-linear curve fits of measured data, error bars are placed at every $0.5 \mathrm{~h}$ for representing standard deviations. e Specific curvature

curvature was observed for all widths. The change of curvature of all bilayer typologies is plotted in Fig. 3b. The highest curvatures were reached by the samples of 20 and $40 \mathrm{~mm}$ width. For $80 \mathrm{~mm}$ and larger widths, the curvature values for all samples were smaller and similar to each other. The rate of change of curvature 
during the first hour after transfer is highest for the samples with $20 \mathrm{~mm}$ width with a value of $2.5 \times 10^{-3} \pm 0.1 \times 10^{-3} \mathrm{~mm}^{-1} \mathrm{~h}^{-1} \quad$ (Fig. 3d), while the rate is well below $2 \times 10^{-3} \mathrm{~mm}^{-1} \mathrm{~h}^{-1}$ for the other bilayers for this time interval. After $2.5 \mathrm{~h}$, however, the rates became very similar for all configurations. The rate of change of curvature was significantly different for the $20 \mathrm{~mm}$ wide sample in respect to the other groups for the first $0.5 \mathrm{~h}$ after transfer.

As moisture content loss was different for the bilayers with different widths at measurement times, specific curvature, defined as the ratio of change of curvature and change of MC, was calculated for each width for revealing the effect of upscaling on the principal behaviour of a bilayer (Fig. 3e). The specific curvatures of the bilayers with different widths were not significantly different within the first $4 \mathrm{~h}$. Between six and $24 \mathrm{~h}$ after transfer, the $240 \mathrm{~mm}$ wide bilayers show a significantly higher specific curvature compared to the 40,80 and $120 \mathrm{~mm}$ wide bilayers. Furthermore, curvatures as a function of MC were calculated using Timoshenko's theory (Online Resource 1-Fig.S3). A linear fit $\left(R^{2}=0.997\right)$ was obtained experimentally and the data correspond well to the calculated curvature. Thus, bilayers of the same configuration, but different width exhibit the same principal behaviour regarding shape change, which facilitates a straight-forward calculation of the shape change of bi-layered lamellae in shading systems.

\subsection{Increasing the rate of moisture change - single desorption phase tests}

The rate of shape change of wood bilayers needs to be sufficiently high for an efficient shading performance. The rate of shape change for a bilayer with a given configuration is directly linked to the rate of moisture change. This rate decreases for increasing widths of wood bilayers as diffusion paths along fibre direction get longer. In the following, we show adaptations in sample surface geometry for increasing the rate of moisture change. Fifteen bilayers of $140 \mathrm{~mm}$ length and $500 \mathrm{~mm}$ width were subjected to desorption for $24 \mathrm{~h}$ by transfer from 85 to $35 \%$ RH. MC loss and change of curvature of all samples were very similar for these two cycles. This procedure was repeated for evaluating reproducibility. After the second desorption phase, ten samples were modified by milling horizontal and diagonal grooves into the beech layer and then put back to $85 \% \mathrm{RH}$ for the re-equilibration. The milling increased the surface to volume ratio of the beech layer of the samples from 0.52 to 0.86 (65\% increase) for the samples with horizontal grooves and to 0.88 (69\% increase) for the samples with the diagonal grooves. At the same time, this milling shortened the length of the diffusion path parallel to fibre direction. All fifteen samples were then exposed to a third phase of desorption at $35 \% \mathrm{RH}$. For the five non-milled samples, a decrease of MC from 18 to $11 \%$ within $24 \mathrm{~h}$ was measured (39\% relative loss). The MC of the milled samples with the horizontal grooves changed from 18 to $9 \%$ (47\% relative loss) after $24 \mathrm{~h}$, whereas a change from 18 to $10 \%$ (45\% relative loss) after $24 \mathrm{~h}$ was recorded for the samples with diagonal grooves (Fig. 4a). In comparison to the non-milled samples, the relative MC losses of the milled samples after $24 \mathrm{~h}$ were higher by $20 \%$ (horizontal configuration) and $15 \%$ (diagonal configuration). The highest rate of MC loss was recorded for the horizontally milled samples during the first hour after transfer, while the non-milled samples showed the lowest rate (Fig. 4d). After $6 \mathrm{~h}$, the rate of MC loss was similar for all configurations. The rate of MC loss was significantly different between the milled and nonmilled samples within the first $3.5 \mathrm{~h}$.

The changes of curvature along the length and the width of the samples are plotted in Fig. 4c. For the first two cycles, very similar curvatures were observed, which proved repeatability for this experiment. Neither in this experiment nor in any other presented experiments visible cracks appeared as signs of excessive stresses in wood or in the bond lines. Regarding the curvature along the length, the samples of both milled configurations showed higher values (increase of 16 and 19\%) compared to those of the non-milled one (Fig. 4c). The rate of change of curvature along the length (Fig. 4e) was highest for the samples with diagonal strips within the first hours of desorption. The rates of the different samples were significantly different from each other for the first 30 min after transfer, while, between 1.5 and $3.5 \mathrm{~h}$, a significant difference was observed between milled and non-milled samples. After $6 \mathrm{~h}$, all three configurations showed similar rates. Furthermore, curvature along the width was evaluated (Fig. 4c) and was found to be one order of magnitude smaller than along the length, which confirms the monoclastic curvature of 
a

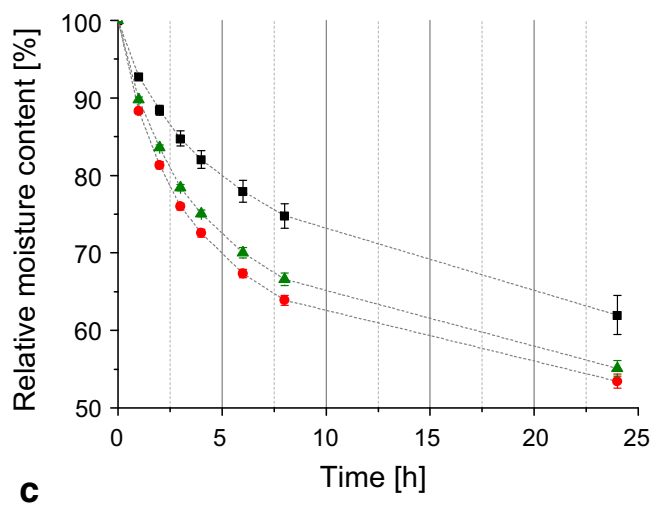

C
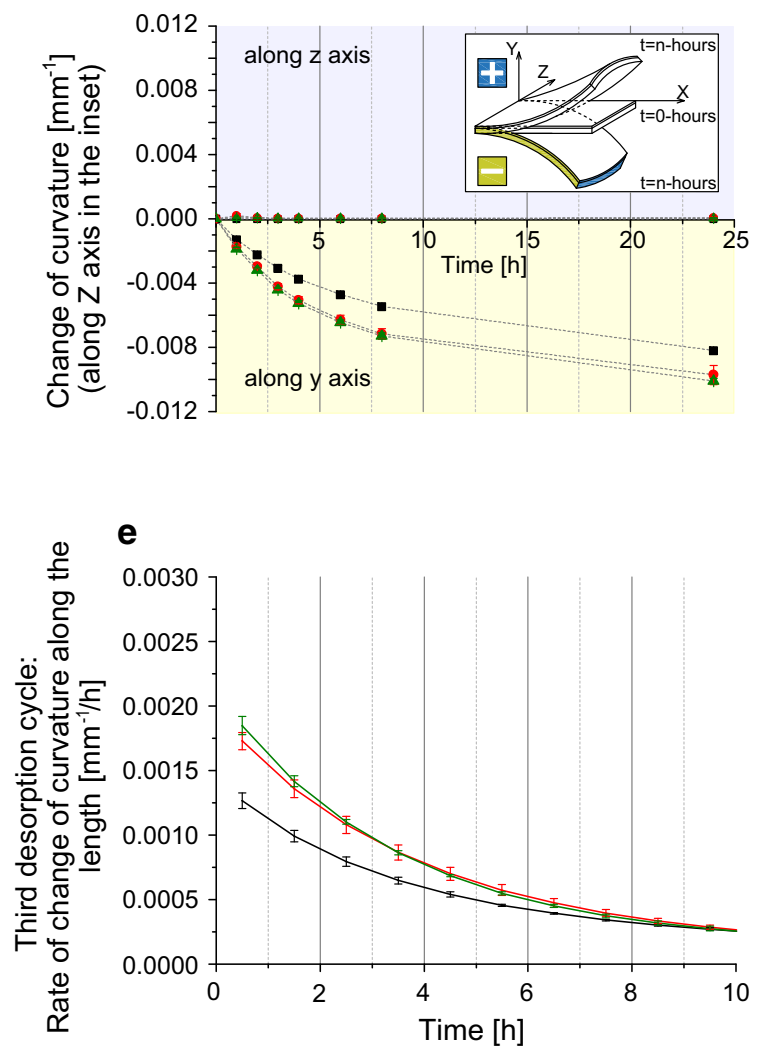

b
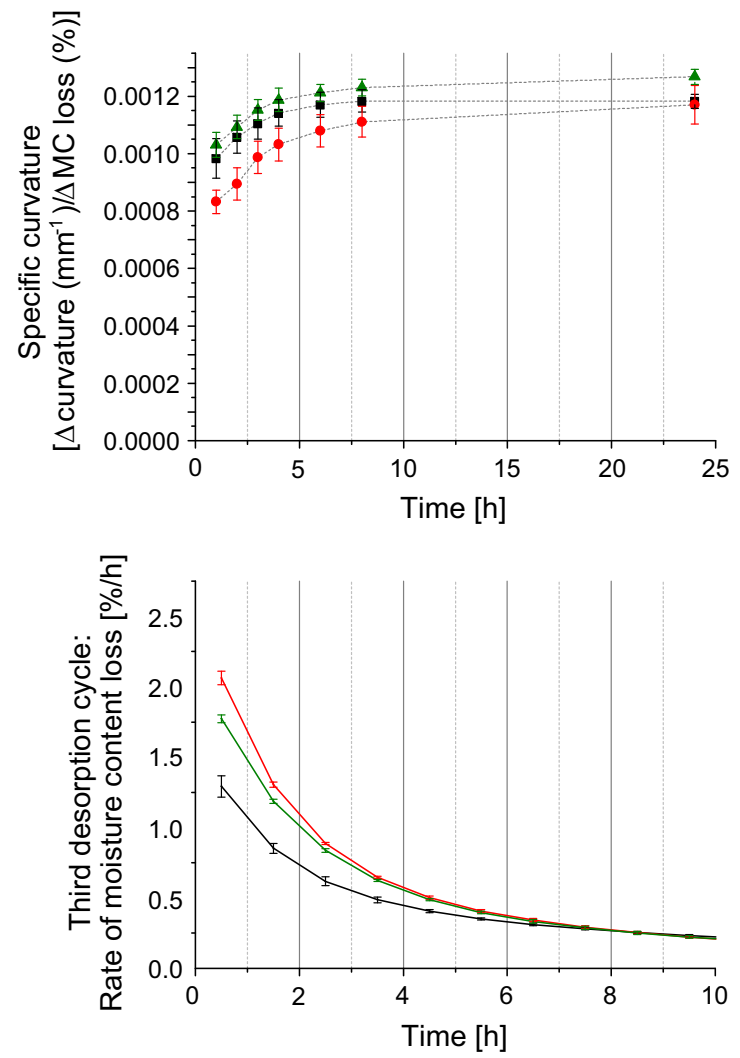

- Third cycle: typology no grooves; - - - Third cycle: typology horizontal grooves; - - Third cycle: typology diagonal grooves;

Fig. 4 Change of MC and curvature of non-milled and milled bilayers after transfer from 85 to $35 \%$ RH. a Relative MC b Specific curvature of the samples during the third desorption cycle after the milling of the grooves c Change of curvature along the length and along the width of the samples after milling the grooves (inset: legend for the sign of curvature along the

the wood bilayers, which had been observed in the former experiments as well.
$Y$ and $Z$ axis) during the third desorption cycle after the milling of the grooves. d Rate of the MC loss of the samples during the third desorption cycle after the milling of the grooves in the first $9.5 \mathrm{~h}$ of desorption e Change of rate of the curvature along the length of the samples for the third desorption cycle after the milling of the grooves in the first $9.5 \mathrm{~h}$ of desorption

The specific curvature, as mentioned in chapter 3.1, is shown in Fig. 4b. The highest values were obtained 
for the samples with diagonal grooves, whereas the samples with horizontal grooves showed the lowest ones. The specific curvature for the samples with horizontal grooves was significantly lower compared to those of the other two configurations within the first $8 \mathrm{~h}$ after transfer. After $48 \mathrm{~h}$, the samples with diagonal grooves showed a significantly higher specific curvature compared to those of the two other configurations.

\subsection{Increasing the rate of moisture change: cyclic change of $\mathrm{RH}$}

For cyclic changes of relative humidity, which resembles the pattern of changes in outdoor application, it is especially beneficial to achieve higher rates of moisture change immediately after the initiation of change. Thus, the response of the modified bilayers to cyclic changes of relative humidity was studied. Three samples from each of the three configurations of the above described investigation were exposed to a cyclic change of RH in a custom-made climate chamber. The RH varied between $15 \%$ and $90 \%$ within each cycle (Fig. S1-c) with the temperature varying between 20 and $22{ }^{\circ} \mathrm{C}$. Successive cycles of $24,12,6,4$ and $2 \mathrm{~h}$ were conducted and repeated four times. Only curvatures along the length were evaluated (Online Resource 1-Fig.S1-b). As the samples were manufactured at $20{ }^{\circ} \mathrm{C}$ and $85 \% \mathrm{RH}$, the curvatures along the length of the samples are either positive (for $\mathrm{RH}>85 \%$ ) or negative (RH $<85 \%$ ) (Fig. 5a: inset). Figure $5 \mathrm{a}$ shows the average curvatures of the three different configurations over time. A movie is available as supplementary online material (Online ressource 2), showing the curvature of the samples during the first $24 \mathrm{~h}$ cycle (see Online Resource 1-Fig.S1-b for an overlay at different times). The curvature values for the three configurations during the $24 \mathrm{~h}$ desoprtion cycle were much higher than those of the previous investigation based on single desorption phases from 85 to $35 \% \mathrm{RH}$ due to the higher differences in $\mathrm{RH}$. Like in the previous experiment, the change in curvature was higher for the samples with the horizontal and diagonal grooves compared to that of the non-milled samples. For each half cycle the amplitude was calculated by taking the difference between the minimum and maximum values of curvature. The amplitude of curvature was higher for the milled configurations compared to that of the nonmilled configuration for all cycles (Fig. 5b). This is valid for all cycle times and all repetitions. In order to further evaluate the level of amplification in the amplitude of curvature as function of the cycle time, the amplitudes of the milled configuration were normalized to that of the non-milled configuration (Online Resource 1-Fig.S1-d). Large variability is visible for the factor of amplification, especially for short cycle times, as the absolute curvatures are low
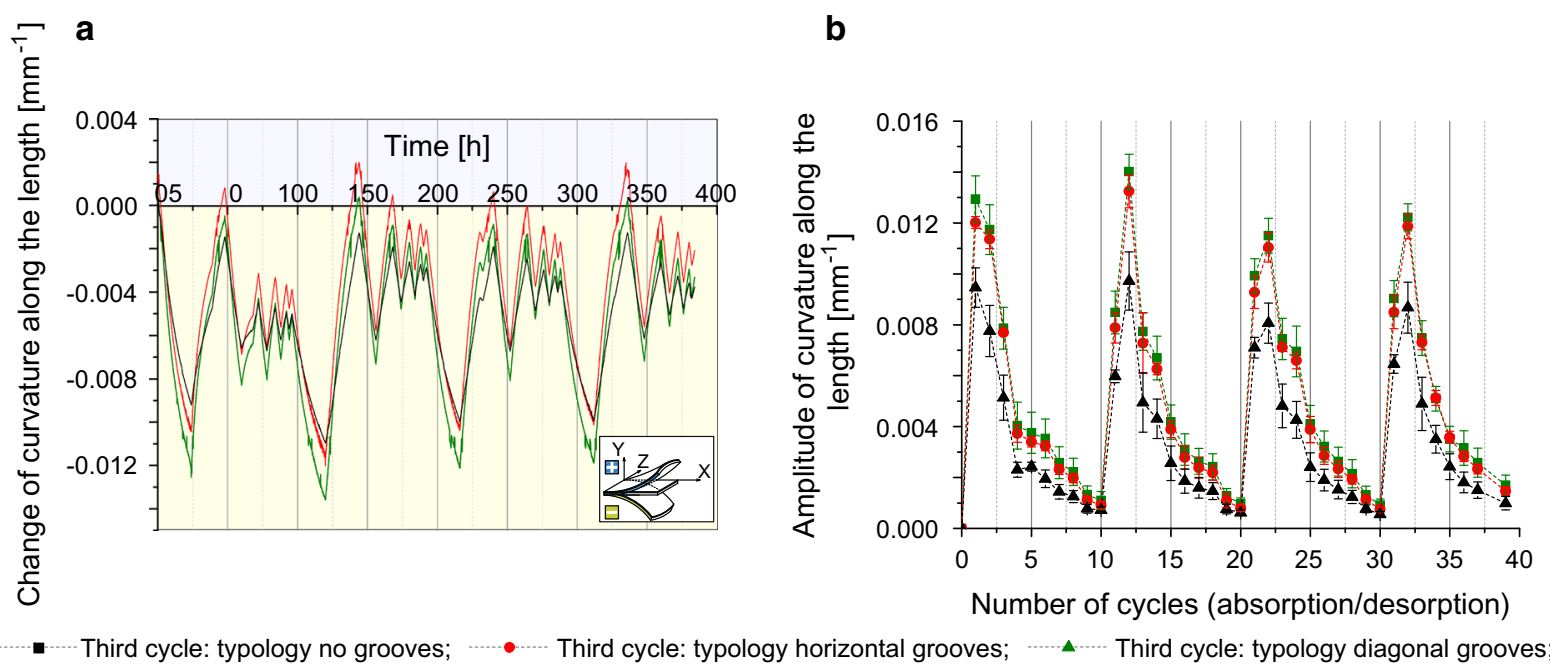

Fig. 5 Curvature of milled and non-milled bilayers in response to cyclic alteration of $\mathrm{RH}$ with varying cycle lengths $(24,12,6$, 4 , and $2 \mathrm{~h}$ ). a Change of curvature along the length for the 4 cycles-sequence (inset: legend for the sign of curvature along the $Y$ axis). b Amplitude of the curvature along the length during the 4 cycles-sequence 
for these cases. A minimum factor of 1.3 is calculated for the cycle time of $24 \mathrm{~h}$ for the horizontal configuration. Amplification factors are higher for shorter cycles with the maximum amplification factor of around 1.6 being visible for the diagonal configuration for cycle times shorter than $6 \mathrm{~h}$.

\subsection{Moisture gradient in the active layer}

For better understanding the effect of the shortening of the diffusion path length on the moisture change rate, desorption experiments were performed with small wood samples with different numbers of surfaces being covered by aluminum foil, which prevents any moisture exchange across this surface (Fig.S2-b; Online Resource 1). Fig.S2-c (Online Resource 1) shows the MC loss until $72 \mathrm{~h}$ after transfer from 85 to $50 \% \mathrm{RH}$. The highest MC loss was observed for the group A with three sides covered, while the lowest MC loss was measured for the group $\mathrm{C}$ with five sides sealed. For group D and group B, both with four sides sealed, but with different configurations, intermediate values were measured. The resulting change in rate of the MC of each group is plotted in Fig.S2-d (Online Resource 1). Within the first $4 \mathrm{~h}$, the rate is highest for group $\mathrm{A}$, and the lowest change was observed for group $\mathrm{C}$.

\section{Discussion}

The responsiveness of wood to changes of $\mathrm{RH}$ can be utilized for novel concepts of autonomous and sustainable climate adaptive building shells. In particular, the implementation of wood bilayers as lamellae for shading systems may lead to energy savings due to reduced cooling needs. Whereas previous investigations focused on curvature predictability and the optimization of the thickness ratio of bilayers [14], the present investigation shows upscaling of the width of bilayers and the optimization of bilayer shape for increasing the rate of moisture change. Both processes are crucial for developing shading systems based on wood bilayers. The upscaling for shading system applications will in most cases require a restriction to monoclastic curvature due to building and fixation geometry. Thus, anticlastic (hyperboloid) curvature, which may occur for wood bilayers with a low aspect ratio, has to be prevented
[24]. Our results show that for the investigated element widths from 20 to $500 \mathrm{~mm}$, a monoclastic curvature could be achieved by using a low thickness ratio of 0.2 for the bilayers. The deformation of the thicker beech layer seems to dominate the entire response of the bilayer, suppressing the bending of the spruce layer perpendicular to fibre direction. Thus, upscaling the width of wood bilayers is facilitated via choosing an asymmetric sample geometry regarding layer thickness.

Next to controlling the pattern of shape change, the rate and amplitude of shape change of such bi-layered lamellae have to be sufficiently high for providing effective shading (and opening of the shading system), as otherwise the lag time of opening and closing would be too large or only partly opening is achieved. Yet, the increase in width of wood bilayers increases the length of the diffusion path of the water along fibre direction, which leads to a reduced rate of MC change. The highest rate of MC loss was measured for the samples with a width of $20 \mathrm{~mm}$ for the first hours after change of RH. This pattern of moisture change rates can be explained by the pronounced anisotropy of diffusion. The diffusion coefficient along fibre direction is more than ten times higher (fifteen times for beech and twelve times for spruce) along fibre direction than perpendicular to fibre direction [25]. Thus, the moisture change rate may be mostly influenced by the length of the element along fibre direction. Thus, samples with lower width (shorter diffusion path along fibre direction) exhibited the highest rate of MC loss. The importance of the diffusion along fibre direction is also shown in the experiment with the cross section sides of the samples differently sealed with aluminum foils (Fig. S2-b). Both, group B and D do not differ in the number of sealed sides (four). However, in group D, the diffusion can only take place in radial direction (from the bond line to the edge of the sample), which is much slower than along the longitudinal direction. Contrary, in group $\mathrm{B}$, only one side perpendicular to the grain is sealed; therefore, the diffusion can occur along the grain and the rate of MC loss was higher for group B than for group D (Online Resource 1-Fig.S2-d).

The effect of sample width on the rate of MC loss is not obvious anymore for widths greater than $40 \mathrm{~mm}$ (as the samples with $480 \mathrm{~mm}$ width showed comparable rates to those with $40 \mathrm{~mm}$ width). This can be explained by the change in the ratio of surface areas 
and the low thickness of the samples. As the two layers are only $0.8 \mathrm{~mm}$ and $4 \mathrm{~mm}$ thick, diffusion across the thickness, even though much lower than that along the grain of the beech layer, becomes dominant for the samples with larger width due to the much larger area of the LR-surface compared to the RT-surface (Fig. 1). Hence, for the present sample geometries, the enhancing effect of diffusion along fibre direction can be seen for the samples with $20 \mathrm{~mm}$ width, but vanishes for larger sample width.

In view of the observed effects and their explanation by the anisotropic diffusion of water in wood, a design strategy was developed for increasing the rate of moisture change for bilayers with large widths as needed for lamellae in large-scale façade shading systems. Grooves were milled into the beech layer at distances of $20 \mathrm{~mm}$ for increasing the rate of $\mathrm{MC}$ change of the bilayers with larger widths. The rate of desorption was largely increased for bilayers with $500 \mathrm{~mm}$ width with the milled grooves in comparison to the non-milled bilayers (Fig. 4d). A slightly higher rate of MC loss was observed for the bilayers with horizontal grooves compared to that of bilayers with diagonal grooves. This can be explained by the fact that the remaining effective width along fibre direction between the grooves was $20 \mathrm{~mm}$ for the horizontal configuration and around $28 \mathrm{~mm}$ for the diagonal configuration (Fig. 2). In general, the increased rate of MC loss of the milled samples is a combination of the reduction of the diffusion path along the grain (Ldirection) of the beech layer and, to a lesser extent, across the grain (along $R$-direction) due to the partial reduction of the thickness of the beech layer to $1 \mathrm{~mm}$.

Because of the substantial increase in the rate of MC loss of the milled samples compared to the nonmilled ones, higher rates of curvature change and, thus, higher overall curvature changes were observed for the milled samples. Hereby, the structural changes introduced to the samples by milling did not lead to any substantial changes in the specific behaviour of the bilayers, as rather similar specific curvatures were calculated for all three configurations. Specific curvature of the samples with horizontal grooves was significantly lower for the first hours after change of $\mathrm{RH}$, but was comparable to the non-milled configuration after $24 \mathrm{~h}$. By then, the specific curvature for the bilayers with diagonal grooves was significantly higher with a difference of around $10 \%$ compared to the non-milled samples. A detailed FE-Model and simulations would be required to better understand the specific behaviour of the three different configurations.

The results of the experiments with a cyclic change of RH correspond well to the above described effects. For all cycles, the milled samples showed higher amplitudes of curvature compared to the non-milled samples. Hereby, the samples with diagonal grooves showed even slightly higher amplitudes of curvature than those with horizontal grooves, which is in contrast to the other experiment, in which both configurations showed very similar changes of curvature. The ratio in amplitudes (milled/non-milled) increases for decreasing cycle times for both configurations. This increase is due to the higher differences in rates of MC loss for the milled and the non-milled samples directly after change of RH than at later stage. Thus, for cyclic changes, especially for those with rather short cycle times, which resemble outdoor conditions more closely, the benefit of the chosen design strategy based on milling grooves is especially visible. However, it cannot be entirely clarified from the two different experiments, whether the configuration with diagonal grooves is even more suitable regarding rate of curvature change than that the one with horizontal grooves. An additional advantage of the configuration with diagonal grooves could be the mechanical stability of the façade elements, as mechanical fortification (due to the remaining parts after milling of the grooves) in diagonal direction is most effective for construction parts.

\section{Conclusion}

Wood bilayers with orthogonal grain orientation show humidity-driven, reversible shape change, which can be utilized for autonomous shading systems. Here, we show how to tackle the challenges of upscaling the size of such wood bilayers. The geometric challenge of avoiding hyperboloid curvature, as both wood layers are swelling and shrinking, is tackled by suppressing the curvature of one layer by introducing asymmetry in layer thickness. With a ratio of layer thickness of 0.2 , we could show monoclastic curvature for bilayer elements with a width of half a meter. Increasing size leads to a lowering of the rate of moisture exchange and, thus, to a lower rate of shape change. By shortening the diffusion path along fibre direction by 
milling grooves into the layer orthogonal to fibre direction, the rate of shape change of bilayer elements with a width of half a meter showed the same rate of shape change than bilayer with a width of $20 \mathrm{~mm}$ (strip geometry). Thus, by developing and employing material adapted design principles, we could show upscaling of wood bilayers while retaining monoclastic curvature and moisture change rate, which facilitates, a wider use of the renewable and $\mathrm{CO}_{2}$-storing resource wood in large-scale, climate adaptive façade shading system.

Acknowledgements C.V. was funded by the Deutsche Forschungsgemeinschaft (DFG) priority program SPP 1420: "Biomimetic Materials Research: Functionality by Hierarchical Structuring of Materials". C.V. and P.H. were funded by the Swiss National Foundation (SNF) Project 163191 "Smart shape-changing wood elements for improved energy efficiency of buildings", which is gratefully acknowledged. The authors would like to thank Thomas Schnider for cutting the spruce and beech samples and Erik Bachtiar for writing the Matlab code for evaluating curvature.

Funding This study was funded by the DFG priority program SPP 1420: "Biomimetic Materials Research: Functionality by Hierarchical Structuring of Materials" and by the SNF Project 163191 "Smart shape-changing wood elements for improved energy efficiency of buildings".

\section{Compliance with ethical standards}

Conflict of interest The authors declare that they have no conflict of interest.

\section{References}

1. Pérez-Lombard L, Ortiz J, Pout C (2008) A review on buildings energy consumption information. Energy Build 40(3):394-398

2. Loonen RC, Trčka M, Cóstola D, Hensen J (2013) Climate adaptive building shells: state-of-the-art and future challenges. Renew Sustain Energy Rev 25:483-493

3. Drake S (2007) The third skin: architecture, technology and environment. UNSW Press, Kensington

4. Tzou H, Lee H-J, Arnold S (2004) Smart materials, precision sensors/actuators, smart structures, and structronic systems. Mech Adv Mater Struct 11(4-5):367-393

5. Carpi F, De Rossi D, Kornbluh R, Pelrine RE, SommerLarsen P (2011) Dielectric elastomers as electromechanical transducers: fundamentals, materials, devices, models and applications of an emerging electroactive polymer technology. Elsevier, Amsterdam

6. Viola EA, Levitsky IA, Euler WB (2010) Kinetics of photoactuation in single wall carbon nanotube-nafion bilayer composite. J Phys Chem C 114(47):20258-20266
7. Erb RM, Sander JS, Grisch R, Studart AR (2013) Selfshaping composites with programmable bioinspired microstructures. Nat Commun 4:1712

8. Fratzl P, Barth FG (2009) Biomaterial systems for mechanosensing and actuation. Nature 462(7272):442-448

9. Burgert I, Fratzl P (2009) Actuation systems in plants as prototypes for bioinspired devices. Philos Trans R Soc Lond A Math Phys and Eng Sci 367(1893):1541-1557

10. Elbaum R, Zaltzman L, Burgert I, Fratzl P (2007) The role of wheat awns in the seed dispersal unit. Science 316(5826): 884-886. https://doi.org/10.1126/science.1140097

11. Dawson C, Vincent JF, Rocca A-M (1997) How pine cones open. Nature 390(6661):668

12. Reyssat E, Mahadevan L (2009) Hygromorphs: from pine cones to biomimetic bilayers. J R Soc Interface 6(39):951-957

13. Le Duigou A, Castro M (2015) Moisture-induced selfshaping flax-reinforced polypropylene biocomposite actuator. Ind Crops Prod 71:1-6

14. Rüggeberg M, Burgert I (2015) Bio-inspired wooden actuators for large scale applications. PLoS ONE 10(4):e0120718. https:// doi.org/10.1371/journal.pone.0120718

15. Timoshenko S (1925) Analysis of bi-metal thermostats. JOSA 11(3):233-255

16. Holstov A, Farmer G, Bridgens B (2017) Sustainable materialisation of responsive architecture. Sustainability. https://doi.org/10.3390/su9030435

17. Reichert S, Menges A, Correa D (2015) Meteorosensitive architecture: biomimetic building skins based on materially embedded and hygroscopically enabled responsiveness. Comput Aided Des 60:50-69. https://doi.org/10.1016/j.cad. 2014.02.010

18. Lisle RJ (2003) Dupin's indicatrix: a tool for quantifying periclinal folds on maps. Geol Mag 140(06):721-726

19. Choong ET, Siau JF (1997) Wood: influence of moisture on physical properties. Wood Fiber Sci 29(3):306

20. Panshin AJ, De Zeeuw C (1980) Textbook of wood technology: structure, identification, properties, and uses of the commercial woods of the United States and Canada, vol 1. McGraw-Hill, New York

21. DIN E 13183-1: Feuchtegehalt eines Stückes Schnittholz Teil 1: Bestimmung durch Darrverfahren; Deutsche Fassung EN 13183-1: 2002. Normenausschuss Holzwirtschaft und Möbel (NHM) im DIN, Berlin 4:34

22. Steiger R, Arnold M (2009) Strength grading of Norway spruce structural timber: revisiting property relationships used in EN 338 classification system. Wood Sci Technol 43(3-4): 259-278. https://doi.org/10.1007/s00226-008-0221-6

23. Ozyhar T, Hering S, Niemz P (2013) Moisture-dependent orthotropic tension-compression asymmetry of wood. Holzforschung 67(4):395-404. https://doi.org/10.1515/hf2012-0089

24. Holstov A, Bridgens B, Farmer G (2015) Hygromorphic materials for sustainable responsive architecture. Constr Build Mater 98:570-582. https://doi.org/10.1016/j.conbuildmat. 2015.08.136

25. Sonderegger W, Vecellio M, Zwicker P, Niemz P (2011) Combined bound water and water vapour diffusion of Norway spruce and European beech in and between the principal anatomical directions. Holzforschung 65(6):819-828 